\title{
ESTIMAÇÃO DO VOLUME DE ÁRVORES UTILIZANDO REDES NEURAIS ARTIFICIAIS ${ }^{1}$
}

\author{
Eric Bastos Gorgens ${ }^{2}$, Helio Garcia Leite ${ }^{3}$, Heleno do Nascimento Santos $^{4}$ e José Marinaldo Gleriani ${ }^{3}$
}

\begin{abstract}
RESUMO - Rede neural artificial consiste em um conjunto de unidades que contêm funções matemáticas, unidas por pesos. As redes são capazes de aprender, mediante modificação dos pesos sinápticos, e generalizar o aprendizado para outros arquivos desconhecidos. O projeto de redes neurais é composto por três etapas: pré-processamento, processamento e, por fim, pós-processamento dos dados. Um dos problemas clássicos que podem ser abordados por redes é a aproximação de funções. Nesse grupo, pode-se incluir a estimação do volume de árvores. Foram utilizados quatro arquiteturas diferentes, cinco pré-processamentos e duas funções de ativação. As redes que se apresentaram estatisticamente iguais aos dados observados também foram analisadas quanto ao resíduo e à distribuição dos volumes e comparadas com a estimação de volume pelo modelo de Schumacher e Hall. As redes neurais formadas por neurônios, cuja função de ativação era exponencial, apresentaram estimativas estatisticamente iguais aos dados observados. As redes treinadas com os dados normalizados pelo método da interpolação linear e equalizados tiveram melhor desempenho na estimação.
\end{abstract}

Palavras-chave: Inteligência artificial, cubagem e árvores individuais.

\section{ESTIMATE OF TREE VOLUME USING ARTIFICIAL NEURAL NETS}

\begin{abstract}
The artificial neural network consists of a set of units containing mathematical functions connected by weights. Such nets are capable of learning by means of synaptic weight modification, generalizing learning for other unknown archives. The neural network project comprises three stages: pre-processing, processing and post-processing of data. One of the classical problems approached by networks is function approximation. Tree volume estimate can be included in this group. Four different architectures, five pre-processings and two activation functions were used. The nets which were statistically similar to the observed data were also analyzed in relation to residue and volume and compared to the volume estimate provided by the Schumacher and Hall equation. The neural nets formed by neurons whose activation function was exponential presented estimates statistically similar to the observed data. The nets trained with the data normalized by the linear interpolation method and equalized presented better estimate performance.
\end{abstract}

Keywords: Artificial intelligence, scaling and tree volume.

\section{INTRODUÇÃO}

Redes neurais artificiais (RNAs) são sistemas massivos e paralelos, compostos por unidades de processamento simples que computam determinadas funções matemáticas (BRAGA et al., 1998). Utilizando um conjunto de exemplos apresentado, as RNAs são capazes de generalizar o conhecimento assimilado para um conjunto de dados desconhecidos. Elas têm, ainda, a capacidade de extrair características não explícitas de um conjunto de informações que lhes são fornecidas como exemplos (KOVÁCS, 1996).

Um projeto de redes neurais consiste em préprocessamento, processamento e, por fim, um pósprocessamento dos dados.

Os problemas tratáveis por meio de redes neurais podem ser divididos em três tipos principais: aproximação

\footnotetext{
${ }^{1}$ Recebido em 13.11.2007 e aceito para publicação em 23.06.2009.

${ }^{2}$ Companhia do Vale do Araguaia. E-mail:<e.gorgens@ gmail.com>.

${ }^{3}$ Departamento de Engenharia Florestal da UFV. E-mail: <hgleite@gmail.com> e <gleriani@ufv.br>.

${ }^{4}$ Departamento de Informática da Universidade Federal de Viçosa da UFV. E-mail: <coord.si@fdvmg.edu.br>.
} 
de função, classificador de padrões e agrupamento de dados (HAYKIN, 2001). Em Ciência Florestal, diversos tópicos podem ser potencialmente tratados por RNAs, entre eles a modelagem do volume de árvores.

Um fato importante ao conduzir inventários florestais é a definição de equações volumétricas apropriadas para cada estrato, sendo este geralmente determinado pelo material genético, pelo espaçamento inicial, pelo regime de corte e pela idade, e, então, geradas equações específicas, atualizadas anualmente. Com isso, empresas de grande porte gastam quantias consideráveis de recursos humanos e financeiros com a derrubada de árvores ao longo do tempo (LEITE e ANDRADE, 2002).

Apesar da contínua atualização de equações volumétricas, em muitas ocasiões não é possível gerar equações para determinados compartimentos. Por exemplo, no caso de plantios clonais e de pesquisa (testes clonais), sempre há estratos onde não é possível derrubar árvores para ajuste de modelos volumétricos, sendo aplicadas equações de outros estratos semelhantes (CAMPOS e LEITE, 2006).

O fato é que, ao longo dos anos, as empresas acumulam grande quantidade de dados de cubagem, incluindo árvores com diferentes características e variações de forma (LEITE et al., 1995). Esses dados podem ser utilizados para implementar um projeto de redes neurais cujo objetivo final é a estimativa do volume de árvores sem o uso de equações volumétricas e de dados específicos de cubagem.

O objetivo deste estudo foi construir uma rede neural que estime com eficiência o volume de árvores.

\section{MATERIAL E MÉTODOS}

Foram coletados dados de cubagem de eucalipto (Eucalyptus spp.) em quatro empresas diferentes, sendo uma no Estado da Bahia, outras duas no Estado de Minas Gerais e a quarta no Estado de São Paulo. Dados de cubagem em teca (Tectona grandis L.f.) no Estado do Mato Grosso também foram utilizados (Tabela 1).

Foi definido como procedimento-padrão o ajuste do modelo de Schumacher e Hall (1933) para cada um desses locais, resultando em cinco equações diferentes.

Foram utilizadas redes anteroalimentadas (feedforward), treinadas por meio do algoritmo da retropropagação do erro (backpropagation). Os dados de entrada para a rede foram: diâmetro a $1,30 \mathrm{~m}$ de altura (dap), altura total (ht), os cinco locais (representados por variáveis binárias) e as duas espécies (também representados pelas variáveis binárias) (Figura 1). Como exemplo, um padrão de entrada para uma árvore de

Tabela 1 - Análise descritiva dos dados usados de cada local. Table 1-Descriptive statistics of the used data per local.

\begin{tabular}{|c|c|c|c|c|c|c|}
\hline$\overline{\text { Local }}$ & Número de árvores & Espécie & Estatística & $\operatorname{Dap}(\mathrm{cm})$ & $\operatorname{Altura}(m)$ & Volume $\left(m^{3}\right)$ \\
\hline \multirow[t]{4}{*}{ SP } & 207 & Eucalyptus spp & Média & 9,71 & 9,09 & 0,0380 \\
\hline & & & Desvio-padrão & 2,51 & 2,00 & 0,0223 \\
\hline & & & Mínimo & 3,00 & 3,40 & 0,0015 \\
\hline & & & Máximo & 15,60 & 13,40 & 0,1094 \\
\hline \multirow[t]{4}{*}{$\mathrm{BA}$} & 164 & Eucalyptus spp & Média & 18,05 & 24,06 & 0,3413 \\
\hline & & & Desvio-padrão & 6,49 & 5,22 & 0,2495 \\
\hline & & & Mínimo & 7,00 & 11,50 & 0,0231 \\
\hline & & & Máximo & 31,00 & 35,00 & 1,0093 \\
\hline \multirow[t]{4}{*}{ MG1 } & 55 & Eucalyptus spp & Média & 24,67 & 27,21 & 0,5664 \\
\hline & & & Desvio-padrão & 5,51 & 6,21 & 0,3098 \\
\hline & & & Mínimo & 16,50 & 16,70 & 0,1472 \\
\hline & & & Máximo & 34,40 & 36,30 & 1,1093 \\
\hline \multirow[t]{4}{*}{ MG2 } & 532 & Eucalyptus spp & Média & 18,15 & 27,31 & 0,4126 \\
\hline & & & Desvio-padrão & 6,29 & 6,14 & 0,3719 \\
\hline & & & Mínimo & 8,65 & 15,78 & 0,0476 \\
\hline & & & Máximo & 37,75 & 47,61 & 2,3597 \\
\hline \multirow[t]{4}{*}{ MT } & 30 & Tectona grandis & Média & 9,87 & 8,06 & 0,0344 \\
\hline & & & Desvio-padrão & 2,87 & 1,90 & 0,0195 \\
\hline & & & Mínimo & 5,70 & 5,10 & 0,0108 \\
\hline & & & Máximo & 15,90 & 12,20 & 0,0766 \\
\hline
\end{tabular}

R. Árvore, Viçosa-MG, v.33, n.6, p.1141-1147, 2009 
eucalipto com dap normalizado de 0,2781 e altura normalizada de 0,3124 no local 5 seria representado pelo vetor $(0,2781,0,3124,0,0,0,0,1,1,0)=(\mathrm{dap}$, ht, L1, L2, L3, L4, L5, E1, E2), em que L1 a L5 representam cada um dos cinco locais e E1 e E2, a espécie. Neurônios de entrada explícitos foram utilizados para garantir representatividade dos cinco locais e das duas espécies.

No pré-processamento, devem ser avaliados a necessidade de aplicar a normalização, a equalização ou, até mesmo, os dois procedimentos aos dados. A normalização é uma técnica de manipulação de banco de dados que visa homogeneizar a grandeza das variáveis envolvidas na análise. A equalização busca ajustar a distribuição dos dados dentro de determinada amplitude (HAYKIN, 2001). As técnicas de normalização e equalização não devem alterar a forma da distribuição dos dados, preservando, assim, as informações implícitas da variável.

No banco de dados utilizados, a existência de variáveis binárias e variáveis contínuas proporciona uma diferença de magnitude entre as variáveis, o que é uma indicação da necessidade de aplicar a normalização.

A solução de RNA proposta neste trabalho utiliza funções de ativação com limites entre 0 e 1 . Equalizar os dados nesse intervalo permite que a rede seja mais sensível à variação desse dados, captando melhor o seu comportamento.

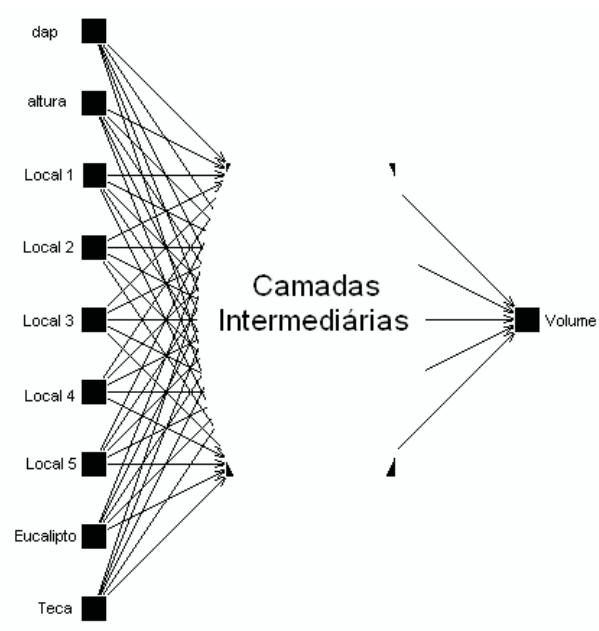

Figura 1 - Esquema com os neurônios de entrada e o neurônio de saída.

Figure 1 - Layout with input and output neurons.
A etapa de pré-processamento foi realizada utilizando diferentes procedimentos encontrados na literatura (GOLDSCHMIDT e PASSOS, 2005).

1) Valor observado da variável (x) dividido pelo valor máximo da variável ( $\left.\mathrm{x}_{\text {máximo }}\right)$.

$$
\text { Valor transformado }=\frac{\mathrm{x}}{\mathrm{x}_{\text {máximo }}}
$$

Esse é um método de normalização em que o valor máximo pode ser obtido por experiência do usuário, por análise do banco de dados ou série histórica.

2) Valor observado da variável ( $x$ ) dividido por um múltiplo de 10, de forma a alterar as casas decimais dos números, para que se incluam no intervalo de 0 a 1 .

$$
\text { Valor transformado }=\frac{x}{10^{n}}
$$

Esse também é apenas um método de normalização, não se preocupando com a equalização dos dados.

3) Interpolação linear inversa entre 0 e 1 , em que x é o valor observado da variável.

$$
\text { Valor transformado }=1+\left(\frac{-x+x_{\text {Minimo }}}{x_{\text {Máximo }}}\right)
$$

A interpolação linear realiza tanto a normalização quanto a equalização. Os processamentos 4 e 5 são variações da forma padrão da interpolação linear.

4) Interpolação linear inversa entre 0 e 0,8 , em que x é o valor observado da variável.

$$
\text { Valor transformado }=0,8+\left(\frac{-x+x_{\text {Minimo }}}{x_{\text {Máximo }}}\right)
$$

5) Interpolação linear entre 0,1 e 0,8, em que x é o valor observado da variável.

$$
\text { Valor transformado }=0,1+\left(0,8 \frac{\mathrm{x}-\mathrm{x}_{\text {Mínimo }}}{\mathrm{x}_{\text {Máximo }}-\mathrm{x}_{\text {Mínimo }}}\right)
$$

Três etapas são necessárias para obter uma rede pronta para ser usada: construção da rede, treinamento e validação. Neste estudo foram avaliadas quatro arquiteturas diferentes.

O algoritmo de aprendizado usado para o treinamento das redes foi o da retropropagação do erro (backpropagation), e os parâmetros de treinamento foram taxa de aprendizagem $(\mu)$ de 0,2 e termo momentum $(\eta)$ de 0,9 . O software utilizado neste trabalho foi o JavaSNNS (ZELL et al., 1998)

R. Árvore, Viçosa-MG, v.33, n.6, p.1141-1147, 2009 
Tabela 2 - Arquiteturas avaliadas para o problema da estimação do volume de árvores.

Table 2 - Evaluated architectures for tree volume estimation.

\begin{tabular}{ccccc}
\hline Arquitetura & 1 & 2 & 3 \\
\hline Função de ativação & Logística & Exponencial & Logística \\
Nodos ocultos 1 & 5 & 5 & 10 & Exponencial \\
Nodos ocultos 2 & 5 & 5 & 10 & 8 \\
\hline
\end{tabular}

Tabela 3 - Identificação das redes de acordo com o préprocessamento e com a arquitetura de rede selecionada.

Table 3 - Neural networks identification: pre-processing versus architecture.

\begin{tabular}{ccccc}
\hline Pré-Processamento & \multicolumn{4}{c}{ Arquitetura } \\
\cline { 2 - 5 } & 1 & 2 & 3 & 4 \\
\hline 1 & 9 & 28 & 29 & 30 \\
3 & 13 & 15 & 16 & 17 \\
4 & 26 & 18 & 19 & 20 \\
5 & 25 & 21 & 22 & 23 \\
\hline
\end{tabular}

(disponível em: <http://www-ra.informatik.unituebingen.de/SNNS $>$ ), e o treinamento da rede se encerrou quando o gráfico do erro tendia à estabilização.

No pós-processamento foi utilizada uma transformação inversa ao pré-processamento, acompanhada de uma regra lógica: "se $X<0$, então $X=0$; caso contrário $X=X$ ”. Tal regra foi importante para assegurar que não ocorressem valores inferiores a zero (realismo biológico).

Os resultados das redes foram comparados com os valores observados e com os estimados pela equação de Schumacher e Hall, por meio do teste t, de gráficos de dispersão XY e de distribuição dos dados.

Aquelas redes que se apresentaram estatisticamente iguais foram analisadas graficamente, sendo as demais descartadas das análises subsequentes.

\section{RESULTADOS E DISCUSSÃO}

Foi utilizada uma equação de Schumacher e Hall (1933) para cada local. Essas equações foram utilizadas para estimar o volume de árvores individuais para os respectivos locais. O conjunto dessas estimativas foi submetido à mesma análise da modelagem por redes neurais.

Diferentemente da tradicional, a modelagem por redes neurais permitiu que todos os locais e espécies fossem modelados por apenas uma rede. As diferentes redes ajustadas foram identificadas de forma numérica, de acordo com a sequência de trabalho, não significando classificação ou preferência (Tabela 3).

As redes 13, 15, 18, 20 e 26 foram estatisticamente iguais aos dados observados diante do teste $\mathrm{t}$ a $5 \%$ de probabilidade. Nas redes 13 e 15 foi aplicado o préprocessamento 3 e nas redes 18, 20 e 26, o préprocessamento 4 . Ambos os pré-processamentos realizam tanto a normalização quanto a equalização dos dados.

Todas as redes que apresentaram resultados estatisticamente iguais aos dados observados foram construídas com duas camadas intermediárias. As redes 13 e 26 exibiram camadas intermediárias com cinco neurônios cada e função de ativação logística. As redes 15 e 18 foram formadas por camadas com cinco neurônios e função de ativação exponencial. A rede 20 possuía camadas intermediárias com 10 e 8 neurônios, respectivamente, e função de ativação exponencial.

Nas redes que apresentaram estimativas semelhantes aos dados observados perante o teste t, uma análise mais profunda foi conduzida para avaliar o comportamento dos dados estimados. Utilizaram-se para tanto gráfico de dispersão observado versus estimado (Figura 2) e gráfico de distribuição dos volumes estimados diante da distribuição real dos dados (Figura 3).

As redes 13 e 18 apresentaram subestimação dos volumes para classes pequenas. A rede 20 mostrou tendência de subestimação geral. A dispersão da rede 26 teve superestimação das classes baixas e subestimação das classes altas.

A avaliação da distribuição é importante para que o processo de estimação mantenha a mesma distribuição dos dados observados, evitando-se, assim, distorções e alterações no comportamento da variável original.

As redes 13 e 26 apresentaram distribuição de diâmetros bastante alterada quando comparada com a dos dados originais. Tal comportamento indica que a estimação do volume pela rede causou distorções dos dados, cuja variável sofreu variação, embora não tenham apresentado diferenças estatísticas pelo teste t. 

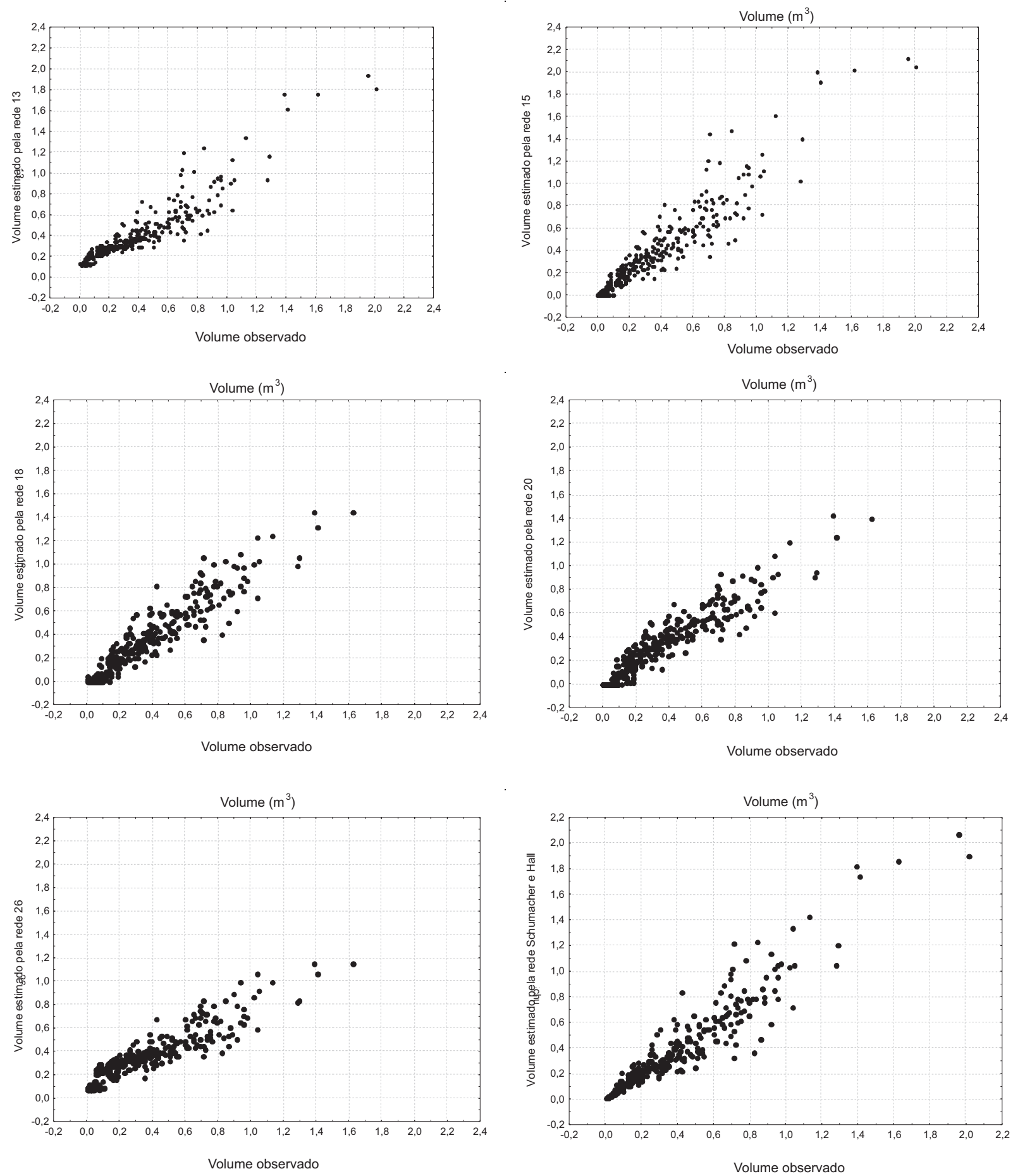

Figura 2 - Valores observados e estimados das diferentes redes em que os resultados foram estatisticamente iguais aos dados observados.

Figure 2-Neural networks where the observed and estimated data are statically similar. 

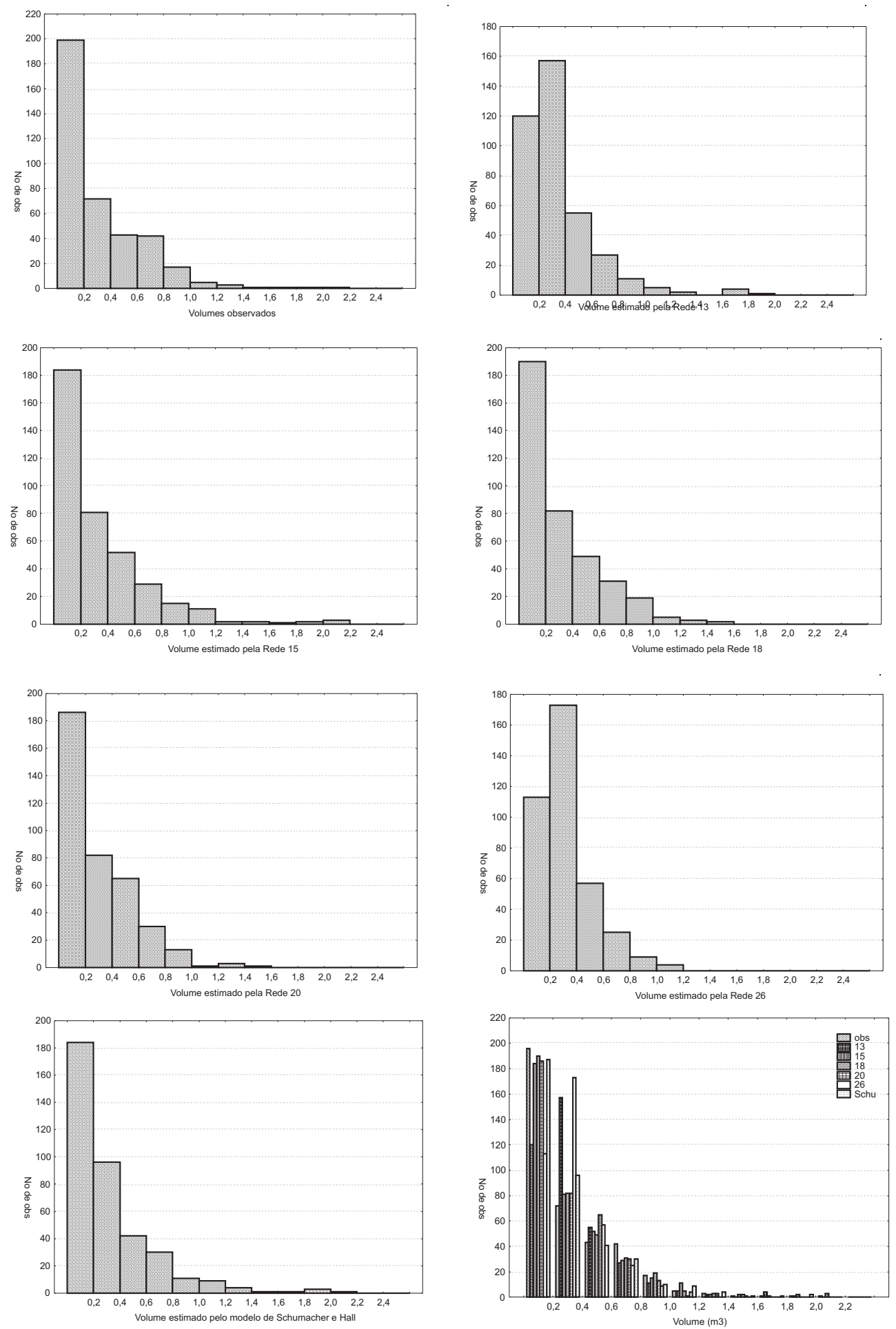

Figura 3 - Distribuição de frequência dos volumes observados e dos volumes estimados pelas redes. Figure 3 - Distribution of observed and estimated volumes.

R. Árvore, Viçosa-MG, v.33, n.6, p.1141-1147, 2009 


\section{CONCLUSÕES}

Diante do exposto, conclui-se que:

- A interpolação linear como método de normalização fornece melhores resultados, e a equalização dos dados melhora a capacidade de predição da rede neural.

- As redes que apresentaram os melhores resultados possuíam duas camadas intermediárias, tendo a função exponencial como "função de ativação". O número de neurônios na camada oculta não resultou em melhorias.

- As redes que apresentaram os melhores resultados receberam como entrada os dados normalizados e equalizados, através de variações do método da interpolação linear.

- A modelagem em rede proporcionou considerável redução na quantidade de equações a serem manipuladas, pois permitiu a abordagem de diversos locais e espécies por uma única rede.

- A modelagem por redes neurais foi perfeitamente viável. Sua capacidade de generalização e conectividade permitiu que se utilizasse apenas uma rede para realizar a predição de volume de árvores dos cinco locais e das duas espécies diferentes.

\section{REFERÊNCIAS}

BRAGA, A. P.; CARVALHO, A. P. L. F.; LUDEMIR, T. B. Fundamentos de redes neurais artificiais. Rio de Janeiro: DCC/I, COPPE/ Sistemas, NCE/UFRJ, 1998. 246p.
CAMPOS, J. C. C.; LEITE, H. G. Mensuração florestal: perguntas e respostas. 2.ed. Viçosa, MG: Universidade Federal de Viçosa, 2006. 470p.

GOLDSCHMIDT, R.; PASSOS, E. Data mining: um guia prático. Rio de Janeiro: Elsevier, 2005. 261p.

HAYKIN, S. Redes neurais: princípios e prática. 2.ed. Porto Alegre: Bookman, 2001. 900p.

KOVACS, Z. L. Redes neurais artificiais: fundamentos e aplicações. 2.ed. São Paulo: Colledium Cognitio, 1996. 174p.

LEITE, H. G.; ANDRADE, V. C. L. Um método para condução de inventários florestais sem o uso de equações volumétricas. Revista Árvore, v.26, n.3, p.321-328, 2002.

LEITE, H. G.; GUIMARÃES, D. P.; CAMPOS, J. C. C. Descrição e emprego de um modelo para estimar múltiplos volumes de árvores. Revista Árvore, v.19, n.1, p.1-21, 1995.

SCHUMACHER, F. X.; HALL, F. S. Logarithmic expression of timber-tree volume. Journal of Agricultural Research, v.47, n.9, p.719-734, 1933.

ZELL, A. et al. SNNS - Stuttgart Neural Network Simulator v.4.2, user manual. Stuttgart: University of Stuttgart/University of Tübingen, 1998. 338p. 
